\title{
Papers
}

\section{Carotid endarterectomy for asymptomatic carotid stenosis:}

a meta-analysis

Oscar Benavente, David Moher, Ba Pham

\begin{abstract}
Objective To assess the value of carotid endarterectomy for prevention of stroke in patients with asymptomatic carotid stenosis.

Design Systematic review and meta-analysis of randomised controlled trials in patients with asymptomatic carotid stenosis in which subjects were allocated to carotid endarterectomy or to medical treatment alone.

Subjects Five trials enrolled 2440 patients with stenosis $\geqslant 50 \%$.

Main outcome measures Stroke ipsilateral to the stenosis, all strokes, and perioperative complications (stroke or death).

Results In patients who underwent carotid endarterectomy $(\mathrm{n}=1215)$ there was a significant reduction in the odds of ipsilateral stroke plus perioperative stroke or death (odds ratio $0.62 ; 95 \%$ confidence interval 0.44 to 0.86 ), corresponding to a $2 \%$ absolute risk reduction over about 3.1 years. The prevalence of stroke in any location was also reduced $(0.68 ; 0.51$ to 0.9$)$ in patients undergoing carotid endarterectomy. During the immediate postoperative period there was an increased prevalence of stroke or death among such patients $(4.51 ; 2.36$ to 8.64$)$.

Conclusion Carotid endarterectomy in patients with asymptomatic carotid stenosis unequivocally reduces the incidence of ipsilateral stroke, though the absolute benefit is relatively small. Given the modest benefit of surgery for unselected patients with asymptomatic carotid artery stenosis carotid endarterectomy cannot be routinely recommended for these patients pending reliable identification of high risk subgroups, and medical management is a sensible alternative for most patients.
\end{abstract}

\section{Introduction}

Asymptomatic carotid stenosis is common, often detected incidentally or in a patient with cervical bruit. Its prevalence ranges from $0.5 \%$ in people under 60 years rising up to $10 \%$ in those over age 80 (an estimated 2 million people in the United States), varying somewhat with the technique of detection and the population. ${ }^{12}$ Patients are at higher risk for ischaemic stroke compared with those without carotid disease, ${ }^{3-5}$ but the risk for stroke is much lower than in patients with symptomatic carotid stenosis. ${ }^{67}$ Patients with asymptomatic carotid stenosis also have a higher risk of vascular death. ${ }^{34}$

Carotid endarterectomy is effective in reducing ipsilateral stroke in patients with symptomatic carotid stenosis with the benefit becoming more evident with greater degrees of stenosis. ${ }^{6}{ }^{7}$ As there are such impressive benefits for those with high grade symptomatic stenosis it has been suggested that carotid endarterectomy could usefully reduce ipsilateral stroke in patients with asymptomatic carotid artery stenosis.

Six randomised clinical trials in patients with asymptomatic carotid stenosis have been conducted. ${ }^{8-13}$ We conducted a meta-analysis to assess quantitatively the efficacy and safety of carotid endarterectomy in this group of patients.

\section{Methods}

Search strategy-We used several strategies to identify all published and unpublished randomised controlled trials that evaluated the efficacy of carotid endarterectomy in patients with asymptomatic carotid stenosis. We completed an electronic search of Medline (OVID) from 1 January 1966 to 30 January 1998 using the following terms: carotid stenosis, endarterectomy carotid, asymptomatic, clinical trial, randomised controlled trial, published in any language. The Cochrane Controlled Trials Register and the Ottawa Stroke Trials Register were also searched. ${ }^{14}{ }^{15}$ The electronic searches were supplemented by hand searching Current Contents from 1 January 1995 to 30 January 1997. Similarly, all issues of the New England Journal of Medicine, Stroke, and the Journal of the American Medical Association were searched from 1 January 1991 to 30 January 1997. In addition, the reference lists of all retrieved articles were reviewed. We also communicated with authorities in this specialty, including the corresponding authors of several of the included trials.

Inclusion criteria - Each trial was evaluated for inclusion in the meta-analysis on the basis of three criteria: study design (randomised controlled trial); target population (patients with asymptomatic carotid stenosis determined by carotid ultrasound or arteriography, either with no history of cerebrovascular disease or with previous stroke or transient ischaemic attack in vertebrobasilar circulation or contralateral carotid territory, and patients who had undergone contralateral carotid endarterectomy); and therapeutic intervention
Editorial by Warlow

Department of Medicine, Division of Neurology, University of Texas Health Science Center, San Antonio, TX 78284-7883, USA

Oscar Benavente, assistant professor

Thomas C

Chalmers Center for Systematic Reviews, Children's Hospital of Eastern Ontario, Ottawa,

Canada

David Moher, director

Ba Pham,

biostatistician

Correspondence to: Dr Benavente

benavente@ uthscsa.edu

BMJ 1998;317:1477-80 
Table 1 Characteristics of randomised clinical trials of endarterectomy for asymptomatic carotid stenosis

\begin{tabular}{|c|c|c|c|c|c|c|c|c|c|}
\hline \multirow[b]{2}{*}{ Study (year) } & \multirow{2}{*}{$\begin{array}{l}\text { No of } \\
\text { patients }\end{array}$} & \multirow{2}{*}{$\begin{array}{l}\text { Proportion } \\
\text { of men }(\%)\end{array}$} & \multirow{2}{*}{$\begin{array}{l}\text { Mean age } \\
\text { (years) }\end{array}$} & \multirow{2}{*}{$\begin{array}{l}\text { Mean } \\
\text { follow up } \\
\text { (years) }\end{array}$} & \multirow{2}{*}{$\begin{array}{c}\text { Degree of } \\
\text { stenosis (\%) }\end{array}$} & \multirow[b]{2}{*}{ Angiogram } & \multicolumn{2}{|c|}{$\begin{array}{l}\text { Antithrombotic treatment } \\
\text { (aspirin mg/day) }\end{array}$} & \multirow{2}{*}{$\begin{array}{l}\text { Quality } \\
\text { score }\end{array}$} \\
\hline & & & & & & & Surgical group & Medical group & \\
\hline \multicolumn{10}{|l|}{ Included trials } \\
\hline Clagett et al $(1984)^{8}$ & 29 & 72 & 64 & 3 & $72^{*}$ & Only surgical group & None & 1300 & 3 \\
\hline MACE (1992) ${ }^{11}$ & 71 & 58 & NR & 2 & $\geqslant 50$ & Only surgical group & None & 80 & 3 \\
\hline VA $(1993)^{10}$ & 444 & 100 & 65 & 4 & $\geqslant 50$ & All patients & 1300 & 1300 & 2 \\
\hline ACAS $(1995)^{12}$ & 1659 & 66 & 67 & 2.7 & $\geqslant 60$ & Only surgical group & 325 & 325 & 3 \\
\hline 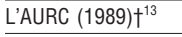 & 237 & 73 & 64 & 5 & $\geqslant 70$ & Only surgical group & 1000 & 1000 & NA \\
\hline \multicolumn{10}{|l|}{ Excluded trials } \\
\hline CASANOVA (1991) ${ }^{9}$ & 410 & 73 & 55 & 3 & $\geqslant 50$ to $\leqslant 90$ & All patients & $975 \ddagger$ & $975 \ddagger$ & NA \\
\hline
\end{tabular}

${ }^{*}$ Mean stenosis.

†Trial completed but not published.

$\ddagger$ Subjects in both groups were also given $225 \mathrm{mg}$ dipyridamole a day.

NR=not reported.

$\mathrm{NA}=$ non-applicable.

See legend to figure 1 for trial names.

(carotid endarterectomy versus or added to medical treatment).

Data extraction - Two authors (OB and DM) selected the trials to be included in the review, extracted the data independently, and cross checked them with disagreements resolved by consensus. The following general descriptive information was extracted from each trial: country or countries in which the trial was conducted; number, age, and sex of participants; degree of stenosis; whether angiography was performed; use of antithrombotic treatment; the number of patients assigned to each intervention; and the number of outcomes in each treatment group. The main outcomes analysed were strokes ipsilateral to the qualifying stenosis combined with all perioperative strokes or deaths; strokes ipsilateral to the qualifying stenosis (including perioperative ipsilateral strokes); all strokes (within and beyond the territory of the qualifying stenosis) combined with all perioperative strokes or deaths; and perioperative strokes or deaths. Perioperative strokes or deaths were defined as those occurring any time after randomisation and extending up to 30 days beyond treatment allocation. All outcomes were counted even if the event occurred before surgery or the patients did not undergo carotid endarterectomy (intention to treat).

Evaluating quality-Included trials were graded independently by two of the authors (OB and $\mathrm{DM}$ ) with a validated three item scale that assigns two points each for describing aspects of randomisation and double blinding and one point for withdrawals and drop outs. The scale ranged from zero to five, with higher scores indicating more complete reporting. ${ }^{16}$

Statistical methods-Estimates of the effectiveness of the intervention were expressed as odds ratios by using a fixed effects model (Peto's method). Heterogeneity between studies was assessed by using the Breslow-Day test of homogeneity. We performed sensitivity analyses including random effects models (DerSimonian and Laird method), trial quality (quality weight), publication status (published versus unpublished), and trial size (largest versus the others). The asymptomatic carotid atherosclerosis study (ACAS) did not report the fraction of the perioperative strokes that were ipsilateral to the operated artery, and we could not get this information from the corresponding author. ${ }^{12}$ Therefore sensitivity analyses were conducted in which perioperative strokes in that study were assumed to be two thirds ipsilateral, all ipsilateral, or none ipsilateral. We evaluated potential publication bias by using the inverted funnel plot approach recommended for meta-analyses with few studies. ${ }^{17}$

\section{Results}

\section{Description of studies}

Six randomised controlled trials were identified for potential inclusion in this meta-analysis (table 1), ${ }^{8-13}$ and five were included. The carotid surgery versus medical therapy in asymptomatic carotid stenosis (CASANOVA) study was not included because a protocol stipulation resulted in nearly half of the patients in the non-surgical group undergoing carotid endarterectomy. Therefore the design of this trial precluded direct comparison of the results with the other studies and did not allow meaningful assessment of the effect of carotid endarterectomy. ${ }^{9}$

Although the French L'AURC trial was completed in 1989, the results have not been fully published, ${ }^{13}$ and the data were obtained and included with the permission of the principal investigator. The four remaining randomised controlled trials were published between 1984 and 1995, and all were reported in English. ${ }^{810-12}$ A total of 1215 patients were randomised to carotid endarterectomy and in 1225 carotid endarterectomy was withheld. The mean follow up varied from 2 to 5 years. Overall there were 7677 patient years representing a mean follow up of 3.1 years. Most $(74 \%)$ patients were men, with the Veterans Administration trial including only men. ${ }^{10}$ The mean age of participants ranged from 64 to 67 years. The degree of carotid stenosis for inclusion ranged from $50 \%$ to $99 \%$, with stenosis initially assessed by Doppler ultrasonography, except in one study which used oculoplethysmography. ${ }^{8}$ In all studies intra-arterial catheter angiography confirmed the severity of stenosis before surgery, but in one trial the method of grading the stenosis was not explained. ${ }^{11}$ The use of antiplatelet agents was advocated for both medically treated patients and those assigned to carotid endarterectomy in the three largest trials. ${ }^{1012} 13$ The Mayo asymptomatic carotid endarterectomy (MACE) trial was terminated prematurely because of high numbers of myocardial infarctions and transient ischaemic attacks in the surgical group, from which aspirin was withheld. ${ }^{11}$ In the Veterans Administration 
cooperative study ${ }^{10}$ and the asymptomatic carotid atherosclerosis surgery study ${ }^{12}$ about one third of the patients had a history of symptoms or carotid endarterectomy on the contralateral side to the qualifying asymptomatic stenosis. Participants in the trial reported by Clagett et $\mathrm{al}^{8}$ and the Mayo asymptomatic carotid endarterectomy study ${ }^{11}$ had no history of cerebrovascular symptoms in any vascular territory. The mean quality score of the trials was 2.75 (SD 0.5) or $55 \%$ of the best quality for trial reporting.

For those allocated to medical treatment without carotid endarterectomy the adjusted prevalence of all stroke or perioperative death was $9.2 \% \quad(95 \%$ confidence interval $7.6 \%$ to $10.8 \%$ ). For the individual studies this figure ranged from $0 \%{ }^{8}{ }^{11}$ to $14.7 \% .{ }^{13}$

\section{Outcome by treatment assignment}

If we consider the constellation of ipsilateral strokes plus all perioperative strokes or deaths the risk among patients allocated to carotid endarterectomy was clearly reduced (odds ratio $0.62 ; 95 \%$ confidence interval 0.44 to 0.86 ; table 2 , figure 1 ), with no significant heterogeneity across the five trials $(P=0.07)$. The adjusted rate of these outcomes was $6.4 \%$ for the medically treated patients, yielding an absolute reduction after carotid endarterectomy of about $2 \%$ over a mean follow up of about 3 years. Sensitivity analyses were conducted with the assumption that, firstly, two thirds of perioperative strokes in the asymptomatic carotid atherosclerosis study occurred around the operated vessel, secondly, all were ipsilateral, and, thirdly, none were ipsilateral; all yielded similar results. Sensitivity analysis that excluded the unpublished French trial also yielded similar results, and analysis by quality weighting did not change these results. An additional sensitivity analysis that excluded the results of the large asymptomatic carotid atherosclerosis study showed very similar reduction in ipsilateral stroke plus perioperative stroke or death $(0.60 ; 0.36$ to 1.01$)$.

When we considered only ipsilateral strokes (including perioperative stroke) as an outcome carotid endarterectomy yielded a significant reduction $(0.46$; 0.32 to 0.66 ; table 2 , figure 1 ) with no significant heterogeneity among the trials $(\mathrm{P}=0.13)$. Sensitivity analyses that excluded the asymptomatic carotid atherosclerosis study and, separately, the French trial yielded similar results.

When we considered all strokes (regardless of vascular territory) plus perioperative death the risk among patients who received carotid endarterectomy was reduced $(0.68 ; 0.51$ to 0.90 ; table 2 , figure 1$)$ with no significant heterogeneity $(\mathrm{P}=0.15)$. Sensitivity analyses that excluded the asymptomatic carotid atherosclerosis study again showed a similar, although non-significant reduction $(0.67 ; 0.42$ to 1.08$)$. Sensitivity analysis that excluded the French trial did not alter the results.

Patients randomised to carotid endarterectomy had a fourfold risk of stroke or death in the 30 days after treatment assignment (4.51; 2.36 to 8.64; table 2, figure 2). In the asymptomatic carotid atherosclerosis study and Veterans Administration trial, several strokes occurred during angiography, and these events were included in this analysis.
Table 2 Effect of carotid endarterectomy on outcomes: results of meta-analysis

\begin{tabular}{llcc} 
Outcome & $\begin{array}{c}\text { Surgical group } \\
\mathbf{( \% )}\end{array}$ & $\begin{array}{c}\text { Medical group } \\
\mathbf{( \% )}\end{array}$ & $\begin{array}{c}\text { Odds ratio* } \\
\text { (95\% Cl) }\end{array}$ \\
\hline Ipsilateral stroke plus perioperative stroke or death & 4.4 & 6.4 & $0.62(0.44$ to 0.86) \\
\hline Ipsilateral stroke plus perioperative ipsilateral stroke & 3.2 & 6.2 & $0.46(0.32$ to 0.66) \\
\hline All stroke plus perioperative stroke or death & 7.4 & 9.2 & $0.68(0.51$ to 0.90) \\
\hline Perioperative stroke or death & 2.4 & 0.4 & $4.51(2.36$ to 8.64$)$ \\
\hline
\end{tabular}

${ }^{*}$ Fixed effect model (Peto's method) was used to derive pooled estimates for rates of ipsilateral stroke, all stroke, and perioperative stroke or death. These rates were calculated by adjusting for different sample sizes across the trials included in the meta-analysis.

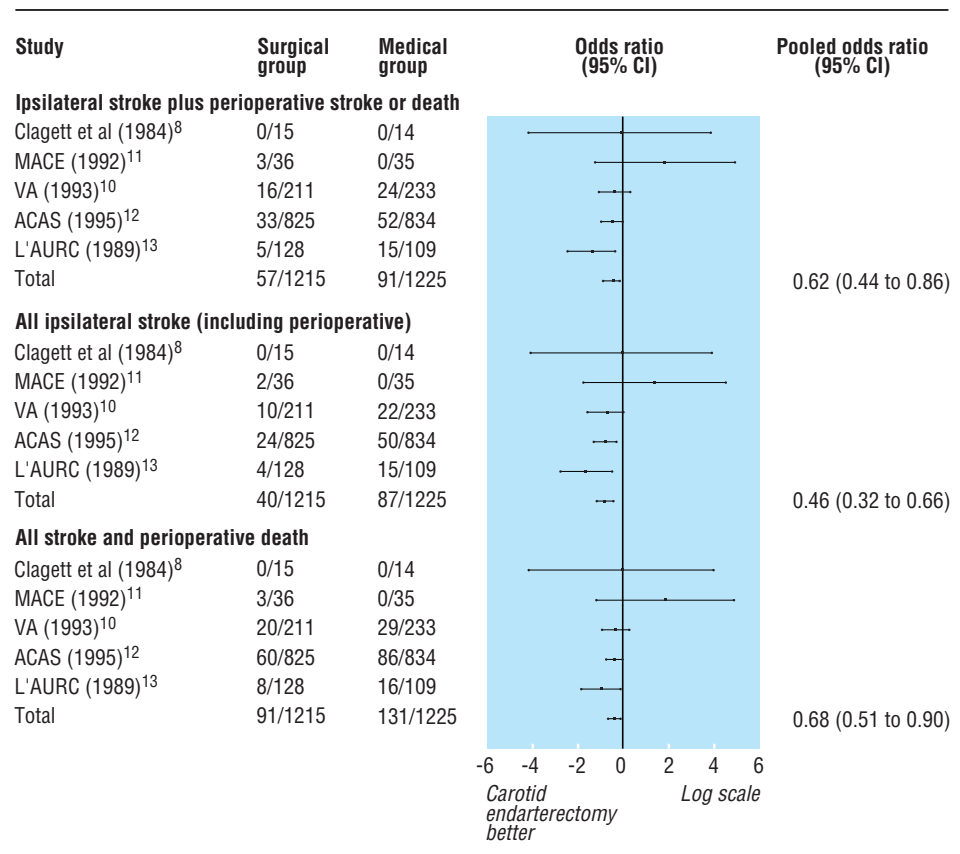

Fig 1 Meta-analysis of aggregated data: effect of carotid endarterectomy in patients with asymptomatic carotid stenosis (MACE: Mayo asymptomatic carotid endarterectomy trial; VA: Veterans Administration trial; ACAS: Asymptomatic carotid atherosclerosis study; L'AURC: Etude multicentique prospective randomisée comparant traitment médical et chirurgical des stenoses serrées atheromateuses asymptomatiques des carotides)

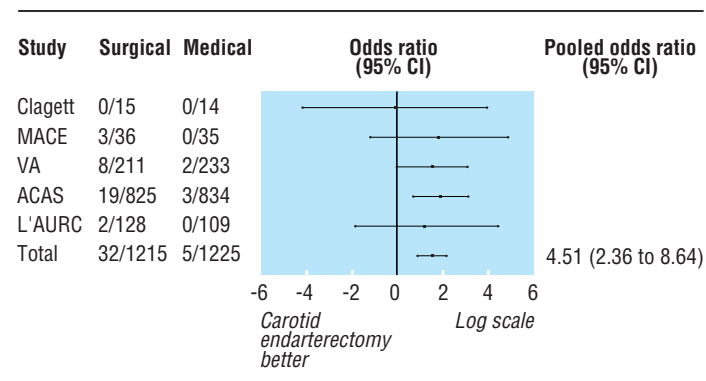

Fig 2 Meta-analysis of perioperative complications (stroke or death) in patients undergoing carotid endarterectomy for asymptomatic carotid stenosis (see figure 1 for abbreviations)

\section{Discussion}

Several clinical trials of carotid endarterectomy for patients with asymptomatic carotid stenosis have been conducted. ${ }^{8-13}$ Important differences among the studies have rendered their interpretation controversial. Two trials were small, with few outcomes and with no ipsilateral strokes. ${ }^{811}$ While all trials included patients with carotid stenosis $\geqslant 50 \%$ the imaging techniques 
mended for unselected patients with asymptomatic carotid stenosis eligible for these trials, despite the substantial reduction in the risk of ipsilateral stroke by surgery. The incidence of ipsilateral stroke was relatively low in those patients who did not undergo the operation and hence the benefit of carotid endarterectomy will remain small until high risk subgroups can be identified.

Contributors: OB designed the protocol, undertook the literature search, discussed core ideas about the study design and interpretation of results, and jointly wrote the manuscript. DM participated in all aspects of the research and jointly wrote the paper. BP completed the computer programming, data analysis, and revisions to the manuscript.

Funding: None

Competing interests: None declared.

used to evaluate the degree of stenosis were not uniform, making direct comparisons difficult.

Despite these issues, the results of this metaanalysis show convincingly that carotid endarterectomy reduces the risk of stroke ipsilateral to asymptomatic carotid stenosis. By using the fail safe method 19 randomised controlled trials of similar sizes with inconclusive results would need to be added to the present analysis to change this conclusion. ${ }^{18}$ It is clear, therefore, that surgical removal of an asymptomatic carotid stenosis definitely reduces subsequent ipsilateral stroke. This magnitude of benefit, however, was not large for patients with asymptomatic carotid stenosis $\geqslant 50 \%$ included in these studies.

Pooled data from these randomised controlled trials showed that the adjusted rate of ipsilateral stroke plus stroke or death at 1 month in the non-surgical group averaged $6.4 \%$ over an average duration of 3.1 years, with an absolute reduction by carotid endarterectomy of about $2 \%$ for the total follow up (table 2). If we consider 3 years of follow up about 50 patients would need to undergo the operation to prevent one event (including disabling or non-disabling stroke).

A major limitation in the current analysis is the lack of uniformity in the measurement of carotid stenosis, potentially resulting in the pooling of subgroups of patients at different risks for stroke. It is conceivable that there are specific subgroups of patients with asymptomatic carotid stenosis who have a high incidence of ipsilateral stroke who would substantially benefit from carotid endarterectomy. At present such a subgroup has not been convincingly identified. A large ongoing randomised trial of carotid endarterectomy for patients with asymptomatic carotid stenosis seeks to resolve these issues. ${ }^{19}$

The results of this meta-analysis suggest that carotid endarterectomy cannot be routinely recom-
1 Ricci S, Flamini OF, Celani MG, Marini M, Antonini D, Bartolini E. Prevalence of internal carotid artery stenosis in subjects older than 49 years: a population study. Cerebrovasc Dis 1991;23:1752-60.

2 Prati P, Vanuzzo D, Casaroli M, Di Chiara A, Di Basi F, Feruglio GA, et al. Prevalence and determinants of carotid atherosclerosis in a general population. Stroke 1992;23:1705-11

3 Meissner I, Wiebers DO, Whisnant JP, O'Fallon M. The natural history of asymptomatic carotid artery occlusive lesions. JAMA 1987;258:2704-7.

4 Norris JW, Zhu CZ, Bornstein NM, Chambers BR. Vascular risk of asymptomatic carotid stenosis. Stroke 1991;22:1485-90.

5 European Carotid Surgery Trialists' Collaborative Group. Risk of stroke in the distribution of an asymptomatic carotid artery. Lancet 1995;345:209-12.

6 North American Symptomatic Carotid Endarterectomy Trial Collaborators. Beneficial effect of carotid endarterectomy in symptomatic patients with high-grade carotid stenosis. N Engl J Med 1991;325:445-53.

7 European Carotid Surgery Trialists' Collaborative Group. MRC European carotid surgery trial: interim results for symptomatic patients with severe $(70-99 \%)$ or with mild $(0-29 \%)$ carotid stenosis. Lancet 1991;337:1235-43.

8 Clagett GP, Youkey JR, Brigham RA, Orecchia PM, Salander JM, Collins GJ, et al. Asymptomatic cervical bruit and abnormal ocular pneumoplethysmography: a prospective study comparing two approaches to management Surgery 1984:96:823-30.

9 CASANOVA Study Group. Carotid surgery versus medical therapy in asymptomatic carotid stenosis. Stroke 1991;22:1229-35.

10 Hobson RW II, Weiss DG, Fields WS, Goldstone J, Moore WS, Towne JB, et al. Efficacy of carotid surgery for asymptomatic carotid stenosis. $N$ Engl J Med 1993;328:221-7.

11 Mayo Asymptomatic Carotid Endarterectomy Study Group. Results of a randomized controlled trial of carotid endarterectomy for asymptomatic carotid stenosis. Mayo Clin Proc 1992;67:513-8.

12 Asymptomatic Carotid Atherosclerosis Study. Endarterectomy for asymptomatic carotid artery stenosis. JAMA 1995;273:1421-8.

13 Lagneau P. Stenoses carotidiennes asymptomatiques. J Mal Vasc 1993;18:209-12.

14 Cochrane Collaboration. The Cochrane Controlled Trials Register. In: The Cochrane Library. Issue 1. Oxford: Update Software, 1998.

15 OSTR Collaborative Group. The Ottawa stroke trials registry collaborative group and the development of the Ottawa stroke trials registry (OSTR). Cont Clin Trials 1994:15:503-11.

16 Jadad A, Moore A, Carrol D, Jenkinson C, Reynolds DJ, Gavaghan DJ, et al. Assessing the quality of reports of randomized controlled trials, is blinding necessary? Cont Clin Trials 1996;17:1-12

17 Begg CB. Publication bias. In: Cooper H, Hedges LV, eds. The handbook of research synthesis. New York: Sage, 1994:399-409.

18 Rosenthal R. The "file drawer problem" and tolerance for null results. Psychol Bull 1979;86:638-41.

19 Halliday AW for the Steering Committee and for the Collaborators. The asymptomatic carotid surgery trial (ACST) rationale and design. Eur J Vasc Surg 1994;8:703-10.

(Accepted 14 August 1998)

\section{Fifty years ago}

\section{The new NHS: Message from the minister}

Perhaps you were almost bound to print the thing, and it may be churlish to complain, but I think that many readers of the Journal will regard "A Message to the Medical Profession" from our Mr Bevan as merely one more, if a minor, irritation. We know that we are supposed to make the best of a rather bad sort of job-a job designed with far too much speed and far too little thought-and I for one do not relish having the matter "rubbed in" by our present Minister of Health, in whom I have hardly any confidence.
It is perfectly obvious that the whole scheme should have been postponed until proper clinics had been built and more nurses trained-a fact which will become very obvious during the next twelve months, the only consolation for which may be that we have some other Minister of Health.-I am, etc, London W4, John C C Langford.

(Letter, 14 August 1948, p 86(suppl). See also editorial by Gordon Macpherson, 3 January 1998, p 6.) 